\title{
Insufficient Anesthesia
}

National Cancer Institute

\section{Source}

National Cancer Institute. Insufficient Anesthesia. NCI Thesaurus. Code C51220.

A state of consciousness that permits the formation of explicit and implicit memories while under general anesthesia. 